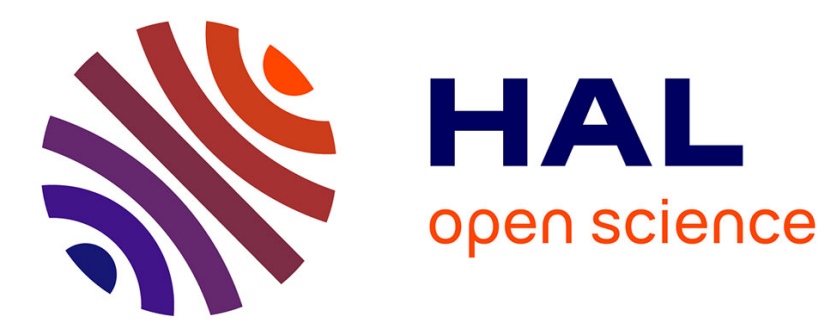

\title{
Le récit du mariage avec la statue. Résurgences et modalités narratives \\ Claudio Galderisi
}

\section{To cite this version:}

Claudio Galderisi. Le récit du mariage avec la statue. Résurgences et modalités narratives. Romania (Paris), 2001, 119 (473), pp.170-195. 10.3406/roma.2001.1552 . halshs-01482118

\section{HAL Id: halshs-01482118 \\ https://shs.hal.science/halshs-01482118}

Submitted on 3 Mar 2017

HAL is a multi-disciplinary open access archive for the deposit and dissemination of scientific research documents, whether they are published or not. The documents may come from teaching and research institutions in France or abroad, or from public or private research centers.
L'archive ouverte pluridisciplinaire HAL, est destinée au dépôt et à la diffusion de documents scientifiques de niveau recherche, publiés ou non, émanant des établissements d'enseignement et de recherche français ou étrangers, des laboratoires publics ou privés. 


\section{Le récit du mariage avec la statue. Résurgences et modalités} narratives

Claudio Galderisi

\section{Citer ce document / Cite this document :}

Galderisi Claudio. Le récit du mariage avec la statue. Résurgences et modalités narratives. In: Romania, tome 119 n473-474, 2001. pp. 170-195;

doi : 10.3406/roma.2001.1552

http://www.persee.fr/doc/roma_0035-8029_2001_num_119_473_1552

Document généré le 16/11/2016 


\title{
LE RÉCIT DU MARIAGE AVEC LA STATUE RÉSURGENCES ET MODALITÉS NAR RATIVES ${ }^{1}$
}

\author{
Pour que l'être soit l'être, il faut que l'Un \\ ne soit pas l'être, mais le générateur de l'être ?2.
}

Le thème du mariage avec la statue peut être inclus et étudié dans celui, plus vaste, des statues vivantes, que l'on retrouve dans toutes les cultures antiques, de la Chine à la Grèce, en passant par l'Égypte ${ }^{3}$. Sa représentation littéraire me paraît, cependant, être exemplaire de la multiplication des identités fictionnelles, et preuve de l'illusoire sur lequel se fonde au Moyen Âge la représentation de l'hic et nunc, du réel quotidien, de l'impensé du corps, mais aussi du conflit mimétique entre corps et esprit ${ }^{4}$.

1. Ce texte reprend et développe une réflexion sur les fonctions sémiotiques des récits du mariage avec la statue que j'ai présentée au colloque de l'Université de Corse du 21 au 23 octobre 1999, à paraître dans Souillure et Pureté. Le corps et son environnement culturel, éd. Jean-Jacques Vincensini, Paris.

2. Plotin, Ennéades, V, $2,1$.

3. Voir, à ce propos, Marie-Claire Weynants-Ronday, Les Statues vivantes, introduction à l'étude des statues égyptiennes, Bruxelles, 1926.

4. Dans un article sur l'altérité et la tenue romanesque dans le Jehan de Saintré d'Antoine de la Sale, j'essayais de réfléchir sur la fonction diégétique d'une statue de cire simulacre du Petit Jehan de Saintré ; une statue que la Dame 
Les sources et les variantes de ce motif, connu depuis longtemps des médiévistes, n'ont jamais été réellement reconstituées et organisées systématiquement. Pourtant, le traitement littéraire de ce motif peut être analysé comme une métaphore de la construction à la fois subjective et traditionnelle - autoscopique et exoscopique - que l'écrivain projette de la structure des actants du récit, à une époque à laquelle le rapport entre auteur et personnage semble soumis à tous les interdits culturels, enfoui entre humilité et anonymat.

Le motif - répertorié par Stith Thompson, qui ne donne, cependant, que deux références ${ }^{s}$, et par Frederic Tubach, qui en signale huit ${ }^{6}$ - semble être particulièrement répandu au Moyen Âge - j'en ai recensé une trentaine pour le Moyen Âge, et plus d'une quarantaine au total - , au point que l'on peut parler d'une véritable diffusion, au sens où l'entendent les diffusionnistes ${ }^{7}$.

des Belles Cousines avait fait construire pour à la fois préserver et piéger l'intégrité psychologique et physique de son protégé. Je soulignais comme le vœu et sa réification investissent l'identité de l'autre, dont la sub-jectivité est de fait piégée, y compris textuellement, dans l'inanité de l'objet, dans la malléabilité protéiforme de la substance cire. L'image de la statue s'offrant, alors, comme une représentation du degré zéro de l'altérité, y compris de l'altérité corporelle, réduite ainsi à un semblant liturgique de réification (voir Claudio Galderisi, "'La femme et le pantin': la statue de cire du Petit Jehan de Saintré, une réification pieuse », colloque du CUER MA, Aix-en-Provence, 5-7 avril 1998, p. 159-173 [Senefiance, 42]).

5. Stith Thompson, Motif-Index of Folk-Literature, Copenhagen, 1956, t. V, p. 354, T. 117.11 .

6. Frederic C. Tubach, Index exemplorum. A handbook of medieval religious tales, Helsinki, 1981, p. $315, \mathrm{n}^{\circ} 4101$ et 4103 . Le $\mathrm{n}^{\circ} 538 \mathrm{du}$ Speculum Laicorum (cf. éd. J.-Th. Welter, Paris, 1914), signalé dans le complément au volume de Tubach, Les Exempla médiévaux. Introduction à la recherche, suivie des tables critiques de l'Index Exemplorum de Frederic $C$. Tubach, sous la direction de Jacques Berlioz et Marie-Anne Polo de Beaulieu, Carcassonne, 1992, est en réalité donné par Tubach sous l'indication Catalogue of Romances in the department of Manuscripts in the British Museum, éd. J. A. Herbert, London, vol. III, 1910, p. 403. La confusion est due sans doute au fait que le système d'indexation du Catalogue... d'Herbert est très complexe.

7. Voir, par exemple, W. A. Clouston, Popular Tales and Fictions. Her Migrations and Transformations, London, 1887, t. II, p. 103-114 et 126-129. Adolf Mussafia a retrouvé la trace de ce récit dans le manuscrit latin 18134 de la 
Voici le résumé d'une des actualisations du récit, d'après l'Histoire de Grégoire VII de Villemain : «On racontait au temps de Grégoire, qu'un jeune romain noble et riche, marié depuis peu, étant allé s'ébattre avec quelques amis sur la vaste place du Colisée, au moment de faire une partie de balle, avait ôté de son doigt son anneau nuptial et l'avait mis au doigt d'une statue de Vénus. Le jeu fini, quand il vint pour reprendre son anneau, il trouva le doigt de marbre de la statue recourbé jusqu'à la paume de la main et il ne put malgré tous ses efforts, ni le briser ni retirer la bague. Il ne dit mot à ses amis et s'en alla fort pensif ; mais il revint une nuit avec un valet. Le doigt de la statue était redressé, mais plus de bague. Rentré dans sa maison, et couché près de sa jeune épouse, il sentit entre elle et lui quelque obstacle palpable mais invisible : et comme il voulait passer outre, une voix lui dit : 'C'est à moi qu'il faut t'unir. Je suis Vénus, c'est à mon doigt que tu as mis l'anneau nuptial: je ne te le rendrai pas'. Le jeune homme, effrayé, trouva toujours entre sa femme et lui le même obstacle. La jeune épouse s'en plaignit à ses parents. Enfin, un prêtre magicien, « Palumbo presbytero, necromantis magno ", ayant contraint Satan à faire rendre l'anneau

Bibliothèque nationale de France (voir Adolf Mussafia, Über die Gautier de Coincy benützen Quellen, in Denkschriften der kaiserlichen Akademie der Wissenschaften. Philosophish-historische Classe, 44, 6, Wien, 1896, p. 35-37). Mussafia est surtout l'auteur d'une étude très riche sur les légendes mariales, dans laquelle il a signalé plusieurs autres attestations du récit (voir A. Mussafia, Studien zu den mittelalterlichen Marienlegenden, t. I, Wien, 1887, p. 48 [962], dans Wissenschaften $\mathrm{n}^{\circ} 29$, p. 72 [986] ; $\mathrm{n}^{\circ}$ 49, dans Sitzungsberichte der kaiserl. Akademie der Wissenschaften in Wien, Philos.-hist. Classe, t. 113, p. 917-994; t. III, Wien, 1889, p. $45, \mathrm{n}^{\circ} 15$, dans Sitzungsberichte der kaiserl. Akademie der Wissenschaften in Wien, Philos.-hist. Classe, t. 119, pars IX). D'autres versions latines ont été signalées par le P. Albert Poncelet, dans Analecta Bollandiana, "Index Miraculorum B. V. Mariae quae saec. VI-XV Latine conscripta sunt ", 21 (1902), p. 241-360, $\mathrm{n}^{\circ} 290$ (repris de Mussafia), 447 (repris de Mussafia), 930 (repris de Bibliotheca Hagiographica Latina, 5371), 938 (repris de N. Van Werveke, Catalogue descriptif des manuscrits de la Bibliothèque de Luxembourg, Luxembourg, 1884, p. 259, XIX, n 20), 1482 (repris de G. Isnard, " Recueil de miracles de la Vierge du XIII" siècle ", dans Bulletin de la Société archéologique, scientifique et littéraire du Vendômois, t. 26, p. 42-44), 1489 (repris de H. L. D. Ward, Catalogue of Romances in the Department of Manuscripts in the British Museum, London, t. II, 1893, p. 626, $\mathrm{n}^{\circ}$ 7), 1490 (tiré de Vincent de Beauvais, Speculum historiale, VII, LXXXVII), 1491 (tiré de Mussafia). 
nuptial par Vénus, le jeune homme fut dès lors heureux sans obstacles ${ }^{8}$ ").

Le texte précise que cette histoire était connue à Rome vers l'an 1046, mais qu'elle remonterait en réalité au $\mathrm{vI}^{\mathrm{e}}$ siècle, à l'époque de saint Grégoire le Grand. Ce récit est le même, à quelques détails près, que celui qui est rapporté par Guillaume de Malmesbury dans les Gesta regum Anglorum ${ }^{9}$. Chez Guillaume de Malmesbury, le prêtre magicien a le même nom « «Palumbo cuidam suburbano presbytero »..., et l'histoire se termine de la même manière : le démon oblige Vénus, défilant comme une prostituée, et fort mécontente, à rendre au jeune homme l'anneau, mais il se venge, en provoquant la mort de Palumbo : "Sed Palumbus, ubi daemonis clamorem ad Deum de se audivit, finem dierum sibi praesignari intellexit ».

Les deux premières sources connues sont sans doute, donc, les Gesta regum Anglorum, et une chronique de Hans Ferdinand Massmann, Der Keiser und der Kunige Buoch, oder die sogennante Kaiserchronik... ${ }^{10}$. Dans cette chronique, composée sans doute à Ratisbonne dans les années 1135-1150, le récit est situé à l'époque de l'empereur Theodosius (vraisemblablement Théodose II), et le protagoniste en est moins le jeune Astrolabius que le prêtre Eusebius. Ici, c'est la statue qui aguiche d'un signe de la main le jeune homme : celui-ci s'en approche et tombe aussitôt amoureux d'elle, en lui promettant un amour éternel. Puis, fatigué de sa situation inconfortable - il ne peut se séparer de la statue - , il se déclare prêt à se convertir au christianisme si un prêtre parvenait à le libérer de l'enchantement. C'est alors qu'un « kâpelan " appelé Eusèbe montre une habileté extraordinaire : il piège le diable auteur du maléfice et l'oblige par un stratagème à lui révéler le secret de l'enchantement. Il apprend alors que ce sont des herbes magiques se trouvant sous le socle de la statue qui retiennent le pauvre Astrolabius. C'est principalement sur ce récit que se fondait la conviction de Gaston Paris d'une origine romaine, remontant au haut Moyen Âge, du motif

8. Abel-François Villemain, Histoire de Grégoire VII, publié par Geneviève Villemain, Paris, 1873 , t. I, p. 272 et suivantes.

9. Guillaume de Malmesbury, «De annulo statuae commendato", dans Gesta regum Anglorum, lib. 11, $\$ 205$, dans J.-P. Migne, Patrologiae cursus completus, Patres Latinis, t. 179, col. 1191, 357.

10. Der Keiser und der Kunige Buoch, oder die sogennante Kaiserchronik..., Quedlinburg und Leipzig, t. II, 1849-1854, p. 264, v. 13102. 
du mariage avec la statue ${ }^{11}$. Une autre attestation se trouve dans le recueil anglais Flores historiarum, commencé vers l'an 1200 par un certain John de Cella, continué par Mathieu Paris et Roger de Wendover 12.

Ce récit, qui comporte de nombreuses variantes ${ }^{13}$, possède, cepen-

11. Voir Gaston Paris, dans Journal des Savants, 1884, p. 567.

12. Roger of Wendover's Flowers of history, trad. John Allen Gilles, Lampeter, t. I, 1995, p. 527.

13. J'ai trouvé d'autres exemples dans les œuvres suivantes : Johann Georg Eckart, Corpus historicum medii aevi, sive scriptores res in orbe universo, praecipue in Germania, a temporibus maxime Caroli $M$. imperatoris usque ad finem saeculi post $C$. n. XV, gestas enarrantes, Lipsiae, t. II, 1723, col. 587-588; Ranulph Higden, Polychronicon Ranulphi Higden..., éd. Churchill Babinton et Joseph Rawson Lumby, trad. John Trevisa, London, 1865-1886, t. VII, p. 220 et suiv. ; Radulphus de Diceto, Abbreviationes chronicorum et imagines historiarum, dans Historiae anglicanae scriptores, éd. J. Seldenus, dans Roger Twysden, Historiae anglicanae scriptores, Londinii, 1652, t. II, col. 471 ; «Sponsalia contrahuntur per anuli tradicionem ", dans Alphabetum narrationum, œuvre qui est attribuée par les uns à Étienne de Besançon, par les autres (en particulier l'érudit anglais J. A. Herbert) à Arnold de Liège. C'est ce récit qui est signalé aussi dans le Speculum Laicorum $\left(\mathrm{n}^{\circ} 538\right)$, tiré à son tour des Gesta regum Anglorum. On le retrouve dans le Catalogue of Romances in the department of Manuscripts in the British Museum, éd. J. A. Herbert, London, vol. III, 1910, p. 403. Ce Catalogue... recense aussi à la p. 437 le récit $n^{\circ} 90$ de l'Alphabetum narrationum, et à la p. $348, n^{\circ} 8$, le conte de la Vie des Pères (A 17), dont il sera question ici. En outre, dans le vol. II, $\mathrm{n}^{\circ} 7$, de ce même Catalogue... est répertorié le récit tiré du Speculum Historiale VII, 87. Tubach (op. cit.) signale aussi Jehan Le Marchant, Le Livre des miracles de Notre Dame de Chartres, écrit en vers, au $X I I I^{e}$ siècle, éd. M. G. Duplessis, Chartres, 1855, p. 127 : « Dou valet anglais qui dona son fermail a liglise de Chartres fere ", ainsi que le recueil catalan Recull de eximplis et miracles e faules e altres ligendes ordenades per $A-B-C$, tretes de un manuscrit en pergami del començament del segle $X V$, ara per primera volta estampades, Barcelona, t. II, 1904, $\mathrm{n}^{\circ} 648$. Mais ce récit n'est autre que la traduction en catalan de celui de l'Alphabetum narrationum. Pietro Toldo (« Dall' Alphabetum narrationum. Gesù e la Vergine ", dans Archiv für das Studium der neueren Sprachen und Literaturen, t. 118 [1907], p. 69-81) étudie les différents récits de l'Alphabetum narrationum consacrés au mariage avec la statue. Il remarque que le $n^{\circ} 648$ est tiré de Guillaume de Malmesbury, et que le $n^{\circ} 649$ est un double du précédent, avec la Vierge Marie à la place de Vénus. De même, le n ${ }^{\circ} 39$ s'avère être une variante du $\mathrm{n}^{\circ} 649$, avec sainte Agnès à la place de la Vierge. La source principale de cette dernière branche est La Légende Dorée. Marcus Landau, "Das Heiratsversprechen", dans Zeitschrift für vergleichende 
dant, une séquence narrative autonome qu'il est possible d'isoler dans

Literatur Geschichte, 1887, p. 19 et 170, propose une autre source, le Leggendario de Bartolommeo da Trento, qui est aussi cité par Arturo Graf, Roma nella memoria e nelle immaginazioni del medio evo, Torino, t. II, 1883, p. 402, où est racontée l'histoire du prêtre Paulinus mettant l'anneau au doigt de l'image de sainte Agnès. La même histoire est racontée dans les Mirabilia Romae e codicibus vaticanis emendata, éd. Gustav Friedrich Constantin Parthey, Berolini, 1869 , p. 98, où il est rapporté que "Sancte Agnetis tenens anulum in manu, quem recipit a presbitero Johanne ex iussu Pascasi ». Arturo Graf signale aussi parmi les sources le Spiegel historiael, qui n'est autre que la traduction faite par Jacob van Marlant du Speculum historiale de Vincent de Beauvais. Pietro Toldo, dans l'article cité, fait aussi référence à d'autres récits qui ont en commun avec le nôtre le don d'un anneau: Étienne de Bourbon, Anecdotes historiques, légendes et apologues, tirés du recueil inédit d'Étienne de Bourbon, dominicain du $X I I I^{e}$ siècle, publiés pour la Société de l'histoire de France, éd. A. Lecoy de La Marche, Paris, 1877, p. 120, ${ }^{\circ} 140$; Jean de Bromyard, Summa praedicantium, Nuremberg, A. Koberger, 1485, p. 117, où un jeune homme met un anneau au simulacre de la Madone "quasi volens eam in uxorem accipere ". L'anneau semble se casser lorsque le jeune homme tombe dans le péché ; ce ne sera qu'une fois qu'il aura fait sa pénitence que l'anneau réapparaîtra intact au doigt de la statue. Dans le Specchio de' prencipi, overo Vite de prencipi santi, ove si leggono varii essempi e usservationi spirituali, morali e politiche del dottore Ranuccio Pico,... - Costantino magno, imperatore, e Guglielmo, duca d' Aquitania, aggiunti ai prencipi santi..., Parma, appresso A. Viotti, 1632-1639, p. 36, on raconte que saint Edmond se consacre à la Vierge, et celle-ci ne voulant pas qu'il ne subsiste pas de trace concrète de tant d'amour, accepte un anneau qui lui est offert par le saint, pour le lui rendre avec le Salut inscrit à l'intérieur. Toldo croit qu'une similarité fonctionnelle entre Vénus et la Vierge peut expliquer aisément ce transfert mythique. Il est vrai que, dans la mythologie, Aphrodite préside aux

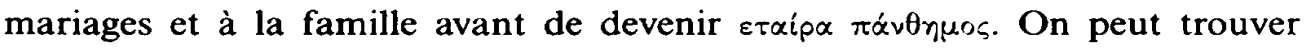
d'autres sources dans Hury of Knighton, dans Twysden, éd. cit., t. II, col. 2335. On peut trouver une bibliographie partielle des différentes sources dans les articles de J. Ulrich, " Miracles de Notre Dame en provençal », dans Romania, t. 8 (1879), p. 12-13, et de Gédéon Huet, " La légende de la statue de Vénus ", dans Revue de l'Histoire des religions, t. 68 (1913), p. 193-217, et surtout dans l'excellent travail, ignoré de presque tous ceux qui se sont intéressés aux sources de ce motif, de P. Franklin Baum, "The Young Man Betrothed to a Statue ", dans Publications of The Modern Language Association, 1919, p. 523-579. Franklin Baum signale aussi deux autres références, dont une que je n'ai pas pu repérer : le Chronicon pontificum de Martin von Troppau. Quant à la seconde, intitulée De annulo triplici, qu'il attribue à Simo Maïolus, il me semble qu'il s'agit en réalité de l'œuvre de Heinrich Kornmann (voir Heinrich Kornmann, 
la chaîne du discours-récit ${ }^{14}$, et qui peut fonctionner à la fois comme un récit autonome et comme partie constitutive d'un récit plus large.

De Annulo triplici : usitato, sponsalitio, signatorio, tractatus absolutissimus, in tres partes divisus, Lugdunum batav., apud Hackios, 1672, p. 41). Ce même Kornmann est aussi l'auteur d'un texte curieux, "De statuis \& miraculis Veneris", «Bon den Statuen, Bildinussen der Göttin Veneris and deren Bunderwerben », in Mons Veneris, Franckfurt am Mayn, J. Fischers, 1614, p. 75-85. Kornmann cite de nombreux exemples d'attraction charnelle pour des statues représentant des femmes (p. 76, « Quintianus : Cedat Praxiteles cuius muliebris imago/Procacem iuvenem impulit ad coitum "), et renvoie à Vincent de Beauvais, Speculum historiale, liv. XXI, cap. $7, \S 4$, où on retrouve le récit de Vénus et de Palumbus, et lib. VII, cap. LXXXVII, « De puero, qui Virginis imaginem anulo desponsavit » - Vincent de Beauvais dit avoir tiré l'histoire « ex Mariali Magno » (le récit est ici situé au temps de Léo IX) - ainsi qu'à $D$. Antoninus, sans aucune autre précision. Cet Antoninus n'est autre que saint Antonin, archevêque de Florence, qui est l'auteur d'une Domini Antonini, archiepiscopi Florentini, chronica, sive opus trium partium historialium, Nurembergae, A. Koberger, t. II, 1484, pars II, titulo XVI, cap. VII, fol. 201-202, §IIII, où est rapportée la même histoire des Gesta regum Anglorum. Voir aussi Aegidius Aurifaber, Speculum exemplorum omnibus christicolis salubriter inspiciendum, ut exemplis discant disciplinam, Hagueneau, Henricum Gran, 1519, distinctio IX, $\mathbf{n}^{\circ}$ XXV ; Pelbartus de Themeswar, Stellarium coronae gloriosissimae virginis..., Venetiis, J. A. Bertanum, 1586, p. 222a, " quarta utilitas", le jeune homme en train de jouer (luderent ad pilam), entre dans une église où il voit une très belle statue de la Vierge... la Vierge apparaît et le jeune homme choisit de suivre la vie religieuse ; Jean Gobi, La Scala Coeli, éd. Marie-Anne Polo de Beaulieu, Paris, 1991, p. 439, n 646 , où on peut lire le même récit que chez Pelbartus, au $n^{\circ} 657$ du même recueil est racontée l'histoire d'un prince fils du roi de Hongrie combattu entre son vœu de chasteté à la Vierge et la nécessité de se marier, qui, après l'apparition de la Vierge au moment de la célébration du mariage, décide de tout abandonner et de devenir ermite près de la ville d'Aquilée en Frioul. Le récit $\mathrm{n}^{\circ} 646$ est tiré par ailleurs du Mariale Magnum, ms. latin 3177 , f. 46-47, I, 36 : « De puero, qui posuit anulum in digito yconie b. Marie... » (voir éd. H. Barre, « L'énigme du Mariale Magnum », dans Ephemerides Mariologicae, t. 16 [1966], p. 265-288, en particulier p. 281). Barre signale aussi que le même récit se trouve dans les manuscrits de Reims, Bibliothèque Municipale 1400 (K 774), ex. $\mathrm{n}^{\circ} 8$, et ms. Add. 15237, ex. $\mathrm{n}^{\circ}$ 7. Marie-Anne Polo de Beaulieu signale aussi parmi les sources possibles la Life of St. Edmund of Canterbury from original Sources, éd. R. P. Wilfrid Wallace, London, 1893, que je n'ai pas pu consulter.

14. Voir, à propos d'une analyse catégorielle des actants du récit, le chapitre que lui a consacré Greimas en 1970 (A. J. Greimas, « La structure des actants du récit ", dans Du sens, Paris, 1970, p. 249-270). 
Voici son unité minimale : un jeune homme en âge de mariage - qui n'est presque jamais doté d'un nom propre, comme c'est souvent le cas dans les contes populaires 15 ,-- ôte son anneau en le mettant au doigt d'une statue de femme, pour pouvoir jouer avec ses amis sans empêchements. Lorsqu'il cherche à le récupérer, la statue ne le lui rend pas, en lui interdisant, explicitement ou indirectement, de s'unir charnellement avec son épouse ou sa future mariée, le don de la bague ayant servi à sceller un pacte d'amour éternel entre la statue et le jeune homme. Dès lors, selon les variantes, on a des catégories narratives et des issues complètement différentes, que l'on peut regrouper selon les trois typologies suivantes :

1) Le jeune homme a recours à un prêtre exorciste, dont il nous est donné de connaître le nom, Palumbus. Celui-ci le libère de l'enchantement, mais au prix de sa propre vie. Ce récit est celui recueilli par les Gesta regum Anglorum de Guillaume de Malmesbury et par le roman en prose du $\mathrm{XIV}^{\mathrm{e}}$ siècle Bérinus ${ }^{16}$; sa diffusion est aussi attestée par un curieux livre à la gloire de Vénus d'un auteur allemand du XVII ${ }^{\mathrm{e}}$

15. Toutefois, dans une famille de manuscrits des Gesta regum Anglorum $\left(A a^{l}, A a^{2}, A a h\right)$, les jeunes mariés sont nommés Lucianus et Eugenia (voir Guillaume de Malmesbury, De Gestis regum anglorum libri cinque, éd. William Stubbs, London, t. I, 1881, p. 256-258). C'est, d'ailleurs, de cette famille, qu'est aussi tiré le récit n ${ }^{\circ} 653$ de l'Alphabetum narrationum (voir An Alphabet of Tales. An english 15th century translation of the Alphabetum narrationum, éd. Mary MacLeod Banks, London, 1904, p. 488-489, n' 730 ).

16. Bérinus, éd. Robert Bossuat, Paris, SATF, 2 vol., 1932-1933, t. I, p. XXVII-XXIX, et t. II, p. 22-35, § 435-444 : « L'Aventure du chevalier Grinus et de la statue à qui il confia son anneau ". Ici, le chevalier se nomme donc Grinus, alors que la statue, elle, est anonyme. Son histoire se termine avec la libération du sortilège par l'œuvre de nécromanciens, après que les prêtres et le pape ont échoué dans leurs exorcismes. Il est intéressant de signaler que l'auteur anonyme de cette mise en prose d'un récit en vers du XIII ${ }^{\mathrm{e}}$ siècle fait précéder le récit de la rencontre entre le jeune homme et la statue par un prologue, où il raconte que : « Il fu jadiz anciennement acoustumé en la cité de Romme, ainçois que en crestienneté fust estoree, que, quant un homme avoit femme ou amie et il advenist qu'elle mourust avant lui, il faisoit faire une ymage a son semblant, le plus proprement que il pouoit; et se l'omme mouroit avant, la femme faisoit faire le semblable » (p. 23). Vœu et prodige païens sont donc associés dans le même récit pour symboliser le mystère de l'altérité immuable : celle de la mort et celle de la pierre. 
siècle, Heinrich Kornmann ${ }^{17}$, ainsi que par le pamphlet de Heinrich Heine, Les Dieux en exil ${ }^{18}$, ou encore par l'ouvrage de Franz von Gaudy, Frau Venus ${ }^{19}$, sans oublier, naturellement, la relecture qu'en propose Richard Wagner dans le Tannhäuser.

Dans ce récit-type, les confins entre paganisme et christianisme sont très lâches, et la perception du corps, ainsi que sa représentation, répond à une double coordonnée épistémologique, étirée entre interdit chrétien et sensualité païenne. Le récit est, de ce fait, en équilibre précaire entre fonction euphorique et dysphorique, selon la perspective du destinataire. Cependant, la statue - qui représente presque toujours Vénus - se transforme ici en opérateur syntaxique, en devenant acteur de discours : "'Ego sum Venus, cujus digito apposuisti annulum ; habeo illum, nec reddam' " ${ }^{20}$, et en défilant, même, avec d'autres divinités païennes dans une procession emmenée par le diable en personne ${ }^{21}$.

17. Heinrich Kornmann, Mons Veneris, op. cit.

18. Heinrich Heine, Les Dieux en exil, Bruxelles, 1853, p. 25-29. Heine dit avoir repris l'histoire du Mons Veneris de Kornmann, mais de l'avoir retrouvée aussi dans un livre bizarre de Delrio, qui l'aurait tirée à son tour d'un ouvrage espagnol. Le Delrio en question est le Père espagnol Martin Anton del Rio, auteur d'un livre en latin sur la sorcellerie, Disquisitionum magicarum..., Moguntiae, J. King, t. II, 1606, p. 148-150, lib. III, sect. IV (traduit et réduit en français quelques années plus tard, avec le titre Les Controverses et recherches magiques de Martino Delrio, Paris, R. Chaudière, 1611), où on retrouve dans le chapitre "De Maleficio ligaminis " la même histoire racontée dans les Gesta regum Anglorum. Dans le livre de Heine, à la différence des Gesta regum Anglorum et de l'Histoire de Grégoire VII, c'est la statue de Vénus elle-même, sans l'intermédiaire donc du Démon, qui obtempère aux ordres contenus dans le parchemin du prêtre, et qui décrète l'arrêt de mort de celui-ci : "Cruel prêtre Palumnus, tu n'es donc pas encore satisfait des maux que tu nous as précédemment infligés! Mais tes persécutions auront bientôt un terme, cruel prêtre Palumnus " (Gesta regum Anglorum, éd. cit., p. 29).

19. Franz Bernhard Heinrich Wilhelm von Gaudy, Frau Venus, dans Sämtliche Werke, éd. Arthur Mueller, Berlin, t. 14, 1844, p. 31 et ss.

20. Guillaume de Malmesbury, Gesta regum Anglorum, éd. cit.

21. Nous avons là un cas très intéressant de confrontation entre trois personnages qui relèvent de trois typologies distinctes de mimésis corporelle : l'actantjeune homme au corps humain, l'actant-démon incarnation de l'esprit (du mal), l'actant-statue reproduisant à la fois l'hic et nunc et l'uchronique du corps humain et son illusion référentielle. 
2) Le jeune homme est ici devenu un diacre - alors que Vénus s'est transformée en Notre-Dame ---, qui quitte sa femme et les biens du monde, et se fait moine, réactualisant dans une perspective toute chrétienne la pratique mythique de la hiérogamie ${ }^{22}$. Cette version est attestée principalement par les différents récits de l'Alphabetum narrationum cités, par un manuscrit éthiopien de la Bibliothèque Nationale contenant une collection de miracles de la vierge, découvert par Hermann Zotenberg ${ }^{23}$, ainsi que par un recueil de miracles ${ }^{24}$, composé par Gautier de Coinci au début du XIII $^{\mathrm{e}}$ siècle $^{25}$. Elle est aussi témoi-

22. Pietro Toldo, dans l'article cité (p. 81), rapporte l'histoire d'Ananda, disciple de Bouddha, qui veut abandonner la divinité pour rejoindre sa fiancée Janapada-Kaliâni, dont il est éperdument amoureux. Bouddha emmène alors le jeune homme au ciel et lui montre des nymphes célestes, en lui demandant si son épouse est plus belle que celles-ci. Le jeune religieux se rend devant autant de splendeur et déclare désormais être prêt à vivre dans la chasteté, à condition qu'après la mort, il lui soit accordé de se joindre à une des ces nymphes célestes. Les railleries de ses compagnons lui feront comprendre la vanité d'un tel désir, et la nécessité de revenir à la chasteté (cf. H. Kern, "Histoire du Bouddhisme dans l'Inde ", dans Revue de l'histoire des religions, t. 5 [1882], p. 164-165.)

23. Voir ms. BNF, nouveau 62, folio 101, publié in Hermann Zotenberg, Manuscrits orientaux de la Bibliothèque Impériale, puis Nationale, Paris, Imprimerie nationale, t. III, «Catalogue des manuscrits éthiopiens, gheez et amhariques de la B. N. ", 1877, miracle $\mathrm{n}^{\circ}$ LXIV. Le manuscrit était connu aussi de Maurice-Louis Vernès, "La Vénus d'Ille de Mérimée et une légende pieuse d'Abyssinie ", dans Revue politique et littéraire, t. 16 (1875), p. 399-401.

24. La fleuraison de recueils de miracles de la Vierge Marie, à la fin du $\mathrm{XII}^{\mathrm{e}}$ siècle et au début du $\mathrm{XIII}^{\mathrm{e}}$ semble être due aussi au désir de l'Église de canaliser le sentiment et le goût populaires pour le miraculeux, en rattachant les prodiges plutôt à une figure semi-divine qu'à des ermites ou des religieux dont la légitimité, et l'existence même, faisait souvent problème.

25. «De l'enfant qui mist l'anel ou doigt l'image Notre Dame», dans Gautier de Coinci, Les Miracles de Nostre Dame, éd. V. F. Koenig, t. II, Genève, 1961 [TLF, 95], p. 197-204. Ce conte a été repris dans des recueils de fabliaux, tels que celui de Barbazan, Fabliaux et contes des poètes françois des XI, XII, $X I I, X I V$ et $X V^{e}$ siècles, tirés des meilleurs auteurs, publiés par Barbazan, nouvelle édition augmentée et revue par M. Méon, Paris, 1808, t. II, p. 420-427, v. 1-196, sous le titre : «Du varlet qui se maria à Nostre Dame, dont ne voult qu'il habitast a autres ", qui compte quinze vers de plus que celui de l'édition Poquet; Legrand d'Aussy, Fabliaux ou contes du XII et XIIT siècles. Fables et romans [sic] du XIIf, traduits ou extraits d'après plusieurs manuscrits du tems..., Paris, 1781 , t. V, p. 55. Ce recueil contient aussi le Lai de l'Ombre, où un 
gnée par deux recueils allemands de légendes mariales de la moitié du $\mathrm{XIX}^{\mathrm{e}}$ siècle ${ }^{26}$, et il en existe aussi une version en provençal ${ }^{27}$.

3) Le jeune homme trouve la mort lors de sa première nuit de noces, sous le poids d'une statue de Vénus qui, à la suite d'un transfert de valeur modale, se déplace, monte des escaliers, aime, embrasse et finalement tue ${ }^{28}$. C'est, bien entendu, l'histoire de la Vénus d'Ille de Mérimée ${ }^{29}$, mais aussi celle de l'opéra Zampa, ou la fiancée de mar-

chevalier s'empare avec la ruse de l'anneau de sa maîtresse, qui s'en aperçoit et cherche à se le faire rendre. Le chevalier décide alors de le rendre à celle qu'il aime le plus

après son amie, et jette l'anneau dans l'eau, à l'image reflétée de l'ombre de la femme.

26. Voir « Marien Bräutigam ", dans Gesamtabenteuer. Hundert altdeutsche Erzählungen : Ritter und Pfaffen-Mären, Stadt und Dorfgeschichten Schwänke, Wundersage- und Legenden..., éd. Friedrich Heinrich von der Hagen, Stuttgart, 1850 , t. III, p. CXXV (bibliographie), et p. 508-511, v. 1-88; "Ein Schüler, Mariens Bräutigam », dans Marienlegenden. Dichtungen des XIIIen Jahrhunderts..., éd. Dr. Franz Pfeiffer, Wien, 1863, p. 53-57, v. 1-88. Les deux textes, pratiquement identiques, semblent être une traduction résumée du récit de Gautier de Coinci. Ils se terminent tous les deux avec le bachelier qui décide de garder sa fidélité à Marie jusqu'à la fin : « Marîen der vrowen sîn des sî gelobt die Kunegîn " (v. 87-88).

27. «En qual maniera un efan clus espozet la ymagina de nostra dona amb un anel e pueis aquest efas lhi mentic ho nolh vole atender sos covenens e nostra dona va lo apelar a son servigi " (Voir J. Ulrich, art. cit., p. 22-23).

28. Marcel Proust, par exemple, en parlant des nouvelles de Mérimée, disait que c'était « le volume où on ne me permettait pas de lire la Vénus d'Ille (voir Contre Sainte-Beuve, éd. Bernard de Fallois, Paris, 1954, p. 238).

29. Prosper Mérimée, Nouvelles, édition, introduction et notes de Michel Crouzet, Paris, t. I, 1987, p. 331-354. Michel Crouzet signale aussi, dans les notes (p. 417-420), d'autres sources possibles, mais non identifiées, à commencer par celles suggérées par Mérimée lui-même, de Freher (lettre datant de 1847 à Éloi Johauneau) et d'un certain Pontanus, que Pierre-Georges Castex croyait impossible d'identifier, étant donné l'ampleur de l'œuvre des divers Pontanus (voir. P.-G. Castex, Le Conte fantastique en France de Nodier à Maupassant, Paris, 1987). Ce Pontanus pourrait être Giovanni Pontano, poète napolitain de la Renaissance, auteur de poèmes scientifiques en latin, qui a écrit un ouvrage, édité par François Rabelais, intitulé Ex reliquis venerandae antiquitatis Lucii cuspidii testamentum, Lugduni, apud Gryphium, 1532. Quant à Freher, il pourrait s'agir de Marquand Freher, Corpus franciscae historiae veteris et sincerae..., Hanoviae, apud haeredes J. Aubrii, 1613, lib. VIII, cap. 15, où il est question 
hre ${ }^{30}$, ou encore d'une comédie en français de Gabriele d'Annunzio,

d'un diacre nommé « Vulficailus ", qui détruit une statue de Diane, et qui reçoit en retour de la déesse d'avoir son corps entièrement recouvert d'ulcères. Francisque Michel avait aussi proposé, dans une lettre à Mérimée, une chronique anglaise composée par un certain "Brompton » (voir Correspondance générale. Lettres à Francisque Michel 1848-1870, dans CEuvres Complètes, éd. Pierre Trahard et Édouard Champion, Paris, t. VI, 1930, p. 40-41, « 10 août 1851 »). $\mathrm{Ce}$ Bromton est un chroniqueur anglais du $\mathrm{Xv}^{\mathrm{e}}$ siècle, qui a écrit un Chronicon Johannis Bromton ab Anno Domini 588 ... 1198, dans Twysden, éd. cit., t. I, col. 950. Tous ces récits sont apparentés au récit-type ${ }^{\circ} 1$, celui des Gesta regum Anglorum. Voir à propos des sources de la Venus d'Ille: Arthur de la Borderie. "La légende originale de la Vénus d'Ille », dans Archives historiques, artistiques et littéraires, t. 1 (1889), p. 492 ; Frank Paul Bowman, "Narrator and Myth in Mérimée's Vénus d'Ille ", dans The French Review, t. 33 (1960), p. 475-482 : G. Lambrechts, "La source immédiate de la Vénus d'Ille », dans Revue de littérature comparée, janvier-mars, t. 42, 1 (1968), p. 110-113; Jean Decottignies, "Quelques rapprochements suggérés par la Vénus d'Ille", dans Revue des Sciences Humaines, fasc. 107 (1962), p. 453-461 ; G. Lambrechts cite parmi les sources possibles Hermann Korner et sa Die "Chronica novella", éd. Jacob Scwalm, Göttingen, 1895, dans laquelle, cependant, je n'ai pu trouver aucune référence à la légende de la statue de Vénus. Il a aussi suggéré un rapprochement avec l'Anatomy of Melancholy de Robert Burton et avec la Venus in Rom de Wilibald Alexis, pseudonyme de Georg Wilhelm Heinrich Häring, écrite en 1828. Michel Crouzet. en revanche, fait référence à Henry James, sans cependant donner d'autres précisions. La nouvelle d'Henry James en question est $L c^{\prime}$ dernier des Valerii (Henry James, The Last of the Valerii, Le Dernier des Valerii, trad. Louise Servicen, préface de Marcel Brion, Paris, 1960, p. 29-57). Ici un jeune noble romain, Valerio, non croyant et intimement païen, découvre dans le jardin de sa villa romaine non pas une Vénus, mais « une Junon » (éd. cit., p. 39), enveloppée d'amples draperies, dont il est immédiatement en adoration, au point d'en délaisser sa femme. Le récit est rapporté par un ami artiste du comte, parrain de sa jeune épouse, qui joue un rôle comparable à celui de l'archéologue parisien -... narrateur de la Vénus d'Ille. Mais, chez James, il n'y a aucun mariage entre le comte et la statue. Junon, d'ailleurs, ne se manifeste ni par des gestes ni par la parole, et son influence est toute esthétique et culturelle. L'adoration que le comte lui voue est celle d'une foi retrouvée : la foi païenne de ses ancêtres. Une fois ce retour aux sources mystiques accompli, la jeune épouse peut faire enterrer à nouveau la statue de Junon, et reprendre sa place aux côtés de son mari.

30. C'est Pierre Jourda qui a signalé le premier ce rapprochement (voir Pierre Jourda, Zampa et la Vénus d'Ille, dans Le Divan, 1945, p. 67-72.) En effet, dans 
La Pisanelle ${ }^{31}$, dans laquelle toutefois la statue de Vénus se contente de faire mourir de frayeur la jeune femme, et de faire perdre sa langue au jeune homme.

Chez Mérimée la statue de bronze de Vénus n'est jamais transformée en acteur syntaxique, exactement comme dans le récit-type $n^{\circ} 2^{32}$, et

la pièce Zampa, ou la fiancée de marbre, représentée la première fois le 3 mai 1831 à l'Opéra-comique (paroles de Anne-Honoré-Joseph Duveyrier, pseudonyme de Mélesville, musique de Ferdinand Hérold, Paris, Riga, 1831, p. 15, I, 13, et p. 30, IV, 9), le brigand Zampa passe une bague au doigt de la statue d'Alice, en prononçant les mots suivants : «Accepte cet anneau, deviens ma femme. Jusqu'à demain je suis à toi ». La statue lui rappellera ce serment à la fin de la pièce, emportant l'homme avec elle au milieu de flammes. C'est le seul cas, avec celui de la Vénus d'Ille, de fiancé tué par la statue, même si l'allusion au Festin de Pierre est évidente, au point que l'auteur lui-même y fait explicitement référence (op. cit., p. 33).

31. Il s'agit d'une pièce composée en français par le vate, intitulée La Pisanelle, qui est un pot-pourri de motifs et de citations médiévaux. Ici, dans le Prologue, le Prince de Tyr, héritier des Lusignan, rapporte au Sire Huguet l'histoire d'un jeune homme nommé Rinier Lanfranc, qui à cause d'un ensorcellement de l'évêque orthodoxe d'Amathonte, lorsqu'il était en train de jouer " au jeu de long-paume ", enleva son anneau, en prononçant la phrase canonique : " femme de cet anneau, je t'épouse ». Seulement, ici, l'apparition de la statue de Vénus, la nuit, produit des résultats surprenants :

"Chaque nuit je viendrai comme dure statue ; mais dans tes bras soudain, de chair serai vêtue. Je viendrai toute blanche comme neige-neigée, et me ferai de rose à la prime gorgée ".

Entre sa "femme simplette et sans mamelles » et les charmes de la déesse de l'amour, le choix ne paraît pas difficile, d'autant plus que sa jeune épouse tombe par terre morte de frayeur. Le jeune Rinier aura pour récompense de perdre sa langue, ce qui est une forme de prétérition fictionnelle. Il est intéressant de remarquer que, comme dans la Vénus d'Ille, chez D'Annunzio aussi, le récit est rapporté par le Prince de Tyr, à qui il a été rapporté par un témoin, puisque Rinier Lanfranc est devenu depuis muet (voir Gabriel d'Annunzio, La Pisanelle ou la mort parfumée, Paris, 1913 ; Tragedie, sogni e misteri, in Tutte le Opere di Gabriele d'Annunzio, éd. Egidio Bianchetto, Milano, t. II, 1980, p. 591-841).

32. En réalité, chez Gautier de Coinci, Notre Dame intervient à deux reprises (v. 121, et v. 142-146), en s'adressant directement au jeune clerc, mais elle le fait lors de deux apparitions en rêve. Prise de parole et matérialité du corps sont donc nettement séparées. Jamais la statue de Notre Dame n'intervient dans le récit à la première personne. 
c'est la jeune mariée-veuve, devenue folle, qui rapporte à des témoins incrédules l'accouplement meurtrier dont elle a été le témoin. Le pacte de lecture est une contrainte, à laquelle l'auteur médiéval semble être moins assujetti que l'écrivain moderne. Le lecteur romantique, à la différence du lecteur médiéval, même en pleine suspension d'incrédulité, veut croire à la salubrité mentale de l'actant-narrateur ${ }^{33}$. Aussi, Méri-

33. Comme l'écrit un commentateur de l'époque : " nos habitudes s'y opposaient, d'une telle sorte que Mérimée a dû, par un artifice ingénieux, mettre le secret du drame dans la bouche d'une folle. Les réflexions infiniment sensées du magistrat qui a recueilli la déposition de la jeune femme sont une précaution narquoise prise par le conteur contre la révolte inévitable du public " (MauriceLouis Vernès, op. cit., p. 239). La question des sources de la nouvelle de Mérimée a passionné les spécialistes de l'écrivain, qui en ont retrouvé un certain nombre. La première référence, suggérée par Mérimée lui-même, est celle au Philopseudès de Lucien, où, toutefois, il n'est question ni d'anneau, ni de mariage, ni, en fin de compte, de statue-femme, puisque l'écrivain de Samosate

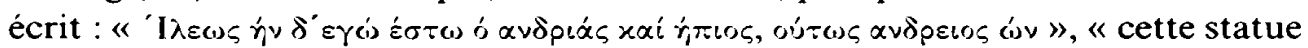
nous soit donc bienveillante et propice puisqu'elle ressemble tant à un homme ", même si elle s'amuse à rosser les gens (voir Lucien, Philopseudès et De Morte Peregrini, éd. Jacques Schwartz, Paris, 1951, 18-19, p. 22). Or en 1837, à part les sources médiévales déjà citées (Giesta regum Anglorum, Kaiserchronik, Gautier de Coinci, etc.) et quelques dizaines de manuscrits de la BNF, Mérimée avait pu déjà avoir eu accès au moins aux deux recueils imprimés de fabliaux et contes cités, qui datent de 1781 et 1808 , et pouvait aussi connaitre l'ouvrage bizarre de Heinrich Kornmann cité, et peut-être aussi le recueil du Père espagnol Martin Anton del Rio, et sans doute l'Histoire de Grégoire VII, publiée en 1873, mais déjà écrite en 1834. Jean Decottignies, dans l'article cité, affirme que Mérimée et Villemain s'étaient rencontrés chez Mme Récamier, en 1834, date à laquelle Villemain venait de terminer son Histoire de Grégoire VII. Une autre source possible de Mérimée se trouve, toujours selon Decottignies, dans un livre bizarre d'Alexis Vincent Charles Berbiguier de Terre-Neuve Du Thym, Les Farfadets ou Tous les démons ne sont pas de l'autre monde, éd. Claude-Louis Combet, Grenoble, 1990, qui n'est autre qu'une reprise de l'histoire racontée par Martin Anton del Rio, comme le prouve la référence à un mystérieux Vincentius Belvacensis, (pour le critique), qui n'est autre que Vincent de Beauvais. Parmi les sources signalées par Decottignies, il y a aussi un traité du Père Costadau, où toutefois je n'ai trouvé des références qu'aux statues de Memnon parlant, et aux simulacres de Junon Moneta et de Fortune (voir Alphonse Costadau, Traité historique et critique des principaux signes qui servent à manifester les pensées ou le Commerce des esprits..., Lyon, frères Bruyset, 1720, t. V, p. 37-39 et 129-131). Mais Mérimée aurait pu être inspiré aussi indirectement, puisqu'il semblerait 
mée est-il obligé de structurer l'armature du mythe, c'est-à-dire le "statut structurel du mythe en tant que narration " ${ }^{34}$, autour d'une série d'enchâssements de discours rapportés : la jeune mariée, sous le choc de la mort de son mari, rapporte ce qu'elle a cru voir au magistrat, qui le rapporte à son tour au narrateur qui le rapporte au lecteur ${ }^{35}$. Le fantastique au $\mathrm{XIX}^{\mathrm{e}}$ siècle, c'est aussi ce jeu de miroirs narratifs qui renvoient la représentation d'un corps de bronze, condamné au silence, qui écrase dans l'imaginaire d'un actant-adjuvant la veuve -mariée, le corps d'un actant- opposant au savoir verbal limité. Comme le suggère Roger Caillois, nous assistons ici à une « lente montée d'une panique infligée à l'esprit le plus rebelle par les mêmes armes qui lui servaient à récuser le surnaturel 36 ". Le récit est construit et écrit sur un mode narratif déceptif. $\grave{A}$ l'intérieur de l'armature du mythe s'opère une inversion : l'actant-statue passe d'un statut d'objet-valeur à un statut de sujet-héros, en même temps que l'actant-narrateur accomplit le parcours inverse. La représentation de l'illusoire au Moyen Âge, tout comme à la fin du $\mathrm{xIX}^{\mathrm{e}}$ siècle, c'est-à-dire aux deux frontières du romanesque, reflète aussi la transformation d'un système de genres

qu'il ne lisait pas l'allemand, par la nouvelle (récente en 1837), de l'écrivain allemand Joseph von Eichendorff, qui avait écrit en 1817 Das Marmorbild, parue posthume. Ici, un jeune italien, Florio, tombe amoureux d'une jeune femme qui n'est autre que la réification a contrario d'une Vénus de marbre anadyomène. Le jeune protagoniste admire cette femme-ombre-statue « comme si la déesse, sortie à l'instant du fond des eaux, contemplait elle-même avec ravissement l'image de sa beauté, que le miroir de l'eau reflétait " (Joseph von Eichendorff, La Statue de marbre, trad. Loeve-Veimars, Paris et Bruxelles, 1943, p. 35). Voir à ce propos Geneviève Bianquis, «Le thème de la Vénus fatale chez Eichendorff et chez Mèrimée ", dans Revue Universitaire (Paris), 1942, p. 85-90, qui cite aussi une autre source possible, Die seltzahme Lucenser Gespeust (Le mystérieux fantôme de Lucques), publié dans Eberhard Werner Happel, E. G. Happelii grösste Denckwürdigkeiten, oder Sogennante relationes curiosae..., Hamburg, T. von Wiering, t. III, 1685, p. 470 : "Die teuffelische Venus"

34. Greimas, op. cit., p. 187.

35. On peut, cependant, remarquer que, dans le récit en provençal cité, où il est d'ailleurs question d'un jeune homme qui joue « al joc de la pilota » (tout comme dans l'ouvrage de Heine), il n'y a aucune prise de parole directe de la part de la statue (à la différence du texte de Heine, mais aussi de tous les autres témoins du récit-type $n^{\circ} 2$ ), et toute la narration est prise en charge par le narrateur.

36. Roger Caillois, Contes étranges, Paris, 1972, p. XII. 
littéraires, la tension entre subjectivité de l'auteur et aséité esthétique des textes, entre intentio auctoris et intentio actoris.

Pour analyser de plus près le fonctionnement sémiotique du récit, j’ai ainsi choisi une variante qui regroupe l'ensemble ou presque des actualisations linguistiques du mythe. Le récit en question, qui occupe 610 vers, est le conte ${ }^{\circ}$ XVII du recueil de la Vie des Pères, intitulé Del fort vallet qui espousa l'ymaige de pierre ${ }^{37}$. L'histoire ne se différencie pas trop au début de celle de la séquence type. Nous sommes au temps de "saint Gregore » (v. 8307), et le jeune homme, appelé ici bourgeois, fraîchement marié, décide de prendre aussi part à un jeu ${ }^{38}$. Cependant, il s'agit ici d'une lutte entre « vaillant bacheler " ${ }^{39}$, et non pas du jeu de la pelote. Ce trait différentiel semble déjà annoncer le conflit fictionnel entre réel et spirituel, la tension entre imaginaire de l'auteur et imaginaire du personnage, sur lesquels se fonde paradoxalement la tenue narrative du conte. D'autant plus que le bourgeois, en ôtant son anneau ${ }^{40}$ et en le mettant au doigt de la statue "en forme de femme entaillée »- - il ne nous est pas donné de savoir s'il s'agit de la statue de Vénus ou d'une autre déesse païenne . , s'amuse à prononcer la formule rituelle du mariage : "Feme, de cest anel t'espous ».

Le récit suit ensuite la trace du schéma-type, jusqu'au moment où le jeune homme cherche à s'unir avec sa jeune mariée. C'est alors que l'" imaige k'il ot espousee " l'empêche matériellement de le faire, en

37. La Vie des Pères, éd. Félix Lecoy, Paris, SATF, t. I, 1987, p. 269-288, v. 8303-8911. Dans le même recueil, on peut trouver un autre exemple de statue vivante, c'est le récit $\mathrm{n}^{\circ}$ LVI, de la seconde Vie des Pères, intitulé "Image de Notre Dame " (La Vie des Pères, éd. Félix Lecoy, Paris, SATF, t. III, 1999. p. 183-196, v. 25086-25497).

38. Quelle que soit la nature de la statue à laquelle le jeune homme met l'anneau, païenne, chrétienne, ou inconnue comme dans le récit de la Vie des Pères, celle-ci semble dès lors vouloir et pouvoir lui insuffler une énergie nouvelle. En effet, dans tous les cas que j'ai pu étudier, le jeune homme l'emporte à tous les coups, et dans différentes sortes de jeux. C'est encore plus évident dans la nouvelle de Mérimée, où le jeune Alphonse semble bien mal en point face au géant espagnol, avant d'enlever sa bague et de remporter la partie de pelote haut la main. Ce qui n'empêche pas, la nuit tombée, la statue de Vénus de le féliciter d'une étreinte fatale.

39. C'est le cas, aussi, du chevalier Grinus dans Bérinus.

40. L'anneau, comme le souligne Jean $\mathbf{R}$. Scheidegger, est un symbole sexuel dans les récits des jongleurs, symbole du désir féminin (voir Jean $\mathbf{R}$. Scheidegger, Le Roman de Renart ou le texte de la dérision, Genève, 1989, p. 315). 
s'interposant entre les deux mariés et en apostrophant rudement le bourgeois :

... Mar i fez !

Tu sez bien ke tu me mesfez

e ke tu m'a hui esposee [...]

totes les fois ke tu voldras

a li gesir, sor toi vendrai

e ton voloir te desfendrai ${ }^{4}$.

Nous avons, donc, une " image ", une statue qui se déplace jusque dans la chambre à coucher du jeune couple, comme dans le récit-type $n^{\circ} 3$, celui de la nouvelle de Mérimée, et qui est acteur de discours, comme dans les récits-type $n^{\circ} 1$, en présence, cependant, d'un deuxième destinataire, la femme de celui-ci, qui voit et entend tout, exactement comme la pauvre «folle » de la Vénus d'Ille, sans cependant mourir de peur comme celle de La Pisanelle. Les pistes narratives sont donc ici brouillées, tout comme le savoir et le pouvoir des personnages, mais tout laisserait à penser qu'à ce moment, le conte est destiné à suivre la pente narrative du récit-type $n^{\circ} 1$, puisque nous savons que la statue représente une déesse païenne, et que c'est la statue même, et non pas son imago, son apparition, qui intervient de tout son poids auprès du bourgeois, pour l'empêcher de s'unir à son épouse humaine. On imagine, à ce moment-là, que la confrontation entre mythologie païenne et mythologie chrétienne va tourner court. En effet, nous savons que, dans le récit-type $n^{\circ} 1$, le bourgeois n'a qu'à s'adresser à un prêtre exorciste, pour que le spirituel chrétien reprenne le dessus sur la réification païenne de l'éternel féminin, délivrant ainsi le bourgeois du sortilège. Et en effet, le bourgeois va voir son chapelain qui, armé de ses parements sacrés, de la croix, du pentacle et de l'eau bénite, se rend dans la maison du couple pour faire un exorcisme contre le prétendu démon.

Dès que les deux époux cherchent à se joindre, la statue se matérialise, mais non seulement elle ne se laisse pas impressionner par les formules du prêtre, par l'eau bénite et par le pentacle, elle fait même la leçon à son époux humain et au prêtre, en les défiant de rompre un lien que la loi de l'homme et de Dieu reconnaît comme indissoluble :

Qui de son bon grei feme espouse si conme cist a moi por voir,

41. Lecoy, éd. cit., p. 273 , v. $8420-8421$ et $8426-8427$. 
bien la doit garder et avoir, por k'il vueille sa loi garder ${ }^{42}$.

C'est au nom de l'institution du mariage et de son indissolubilité ${ }^{43}$ spirituelle et physique, ainsi que contractuelle ${ }^{44}$, que la statue, donc, réclame le respect de l'engagement auquel l'actant-bourgeois, qui n'a pas la chance de connaître le grec, ne pourra pas répondre à la manière

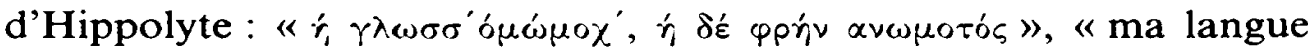
prêta serment, mais non pas mon cœur " ${ }^{45}$. Cependant, l'actantstatue revendique aussi le respect d'un énoncé contractuel. La formule prononcée par le bourgeois n'est pas simplement une suite de mots, parce que le mariage, c'est justement « dire quelques mots bien déterminés " ${ }^{46}$, c'est un énoncé performatif, peut-être le seul véritable, qui transforme une proposition verbale en réalité factuelle, le verbe en chair - selon Tertullien, la chair est le « gond du salut » («caro salutis est cardo ») - , à condition, cependant, qu'il soit prononcé dans des circonstances appropriées. Or, c'est exactement sur la nature à la fois appropriée et inappropriée de ces circonstances que se fonde le malentendu d'où le conte tire son origine. Deux épistémè, la païenne et la chrétienne, sont ici posées in praesentia.

Les deux actants sujet-héros et objet-valeur sont dotés de deux savoirs

42. Ibid., p. 275 , v. $8489-8492$.

43. Comment ne pas rappeler que c'est justement vers la seconde moitié du $\mathrm{XI}^{\mathrm{e}}$ siècle que l'église commence à consacrer une attention nouvelle à l'institution du mariage, avec une série de decreta qui tendent à remplacer la pratique semi-païenne de la bénédiction des époux, avec le sacrement du mariage (voir, par exemple, à ce propos, Georges Duby, Le Chevalier, la Femme et le Prêtre, Paris, 1981, chap. III).

44. Arturo Graf faisait observer à ce propos que le jeune homme accomplit un acte imprudent, constituant un symbole juridique, et qu'il est devenu de ce fait le mari de la statue, qui dès lors réclame ses droits d'épouse. La non démonisation de la figure de Vénus fait d'ailleurs pencher l'historien italien (rejoint sur ce point par Gédéon Huet, art. cit.) pour une formation relativement tardive du récit (1000-1050), à une époque où le conflit religieux entre paganisme et christianisme a été définitivement remporté par ce dernier (voir Arturo Graf, op. cit., Torino, t. II, 1883, p. 388-402). En effet, le malentendu fictionnel se fonde sur un brouillage des deux codes épistémologiques.

45. Euripide, Hippolyte, éd. Louis Méridien, Paris, t. II, p. 52.

46. J. L. Austin, How to do Things with Words, Oxford, 1962, éd. franç., Quand dire, c'est faire. Première conférence, trad. Gilles Lane, Paris, 1970, p. 45. 
encyclopédiques conflictuels. Et ce sont ces deux conceptions du mariage, mais aussi du "grand malentendu des sexes " ${ }^{47}$, selon la formule de Jankélévitch, qui restructurent par leur tension le motif mythique, le remythisent ${ }^{48}$, en même temps qu'ils redéfinissent la structure narrative, l'armature du récit. Ainsi, l'actant-bourgeois, d'après sa croyance de l'état de choses du monde - le savoir dont l'auteur l'a doté -, croit que la circonstance de son engagement est inappropriée ${ }^{49}$, alors que l'actant-statue croit - ou doit croire, dans sa fonction d'opposant - exactement l'inverse. En prononçant, donc, cette phrase apparemment anodine, l'actant-bourgeois n'inscrit pas seulement son personnage dans une fiction, il en crée en définitive les présupposés romanesques. Par son caractère performatif, le mariage constitue un acte antilangagier, factuel, foncièrement, donc, antiromanesque. On le sait, le mariage fictionnel n'intervient qu'à la fin du récit, pour signifier au lecteur la fin du pacte de lecture, selon la formule traditionnelle : "et ils vécurent heureux... ". Il marque la fin du récit, en laissant généralement les actants-époux aux prises avec le silence du lecteur, et les « pépins » de leur vie quotidienne. Alors qu'ici, l'acte de parole qui scelle cette union impossible marque le début de la narration, et les malheurs du personnage que l'actant interprète : les malheurs d'un bigame condamné à l'abstinence. Cependant, l'actantbourgeois semble savoir, sur un plan métafictionnel, que sa vie d'honnête mari aux côtés de son épouse « simplete et jone sanz mamele » ne possède pas -- ou pas encore - de dignité romanesque. Du coup, le sien n'est pas un acte manqué, mais plutôt un acte métaromanesque parfaitement réussi.

47. Voir Vladimir Jankélévitch, Le Je-ne-sais-quoi et le Presque-rien. 2. La méconnaissance. Le malentendu, Paris, 1980, p. 203.

48. Au sens où l'entend Paul Ricœur, qui attribue à la seule réalité fictionnelle, artistique, le pouvoir d'évoquer et faire coïncider vision mythique du temps et actualisation de remythisation (cf. Paul Ricœur, Temps et récit. 3. Le temps raconté, Paris, 1985, p. 238).

49. À ma connaissance, les critiques qui se sont intéressés à la mise en écriture de ce motif mythique n'ont pas remarqué que, dans les récits-type $n^{\circ} 2$, le jeune homme met au doigt de la statue non pas la bague qu'il a destinée à son épouse, mais une bague qui lui a été vraisemblablement offerte par celle-ci, simplement parce qu'il est déjà marié. Le jeune homme se retrouve dès lors dans une position de bigame, ce qui peut expliquer aussi les sept ans d'expiation auxquels il est condamné dans le conte de la Vie des Pères. 
Mais l'actant-statue, aussi, affirme ses droits à la fois fictionnels et métaromanesques, en imposant une présence physique, corporelle double mécanique de l'être vivant $50 \ldots$, que les autres personnages n'ont ni dans le monde possible de la fiction, ni dans le monde réel du lectcur. Et puisque les personnages ont sur elle au moins un avantage apparent, l'acte de parole, la pierre aussi se fait verbe ${ }^{51}$. Et son acte de parole est d'autant plus fort que cette lettre devient ipso facto littéraire, au sens étymologique d'inscription de la parole, mais aussi au sens d'une distorsion de la mimésis, car, on le sait, dans le monde du lecteur, les statues ne parlent pas. Sa parole est d'emblée littéraire - que l'on pense à la statue du Commandeur dans Don Juan - parce qu'elle est gravée dans la mémoire ineffaçable du marbre, dans la tenue ontologique d'un corps et d'une conscience soudés dans l'illusion référentielle de la pierre, dans la vérité absolue du mythe.

Commence, alors, toute une série de péripéties narratives, que je résume brièvement. Le jeune couple va d'abord voir le pape, auquel il raconte son aventure. Celui-ci, inquiet de savoir l'échec du pouvoir religieux connu de tous, conseille aux deux l'abstinence sexuelle ${ }^{52}$. Le bourgeois obéit à contrecœur .... le texte ne nous dit rien de la jeune femme -, mais cherche tout de même un autre avis auprès d'un célèbre ermitc, qui lui conseille de continuer son régime d'abstinence, mais surtout de prévoir un service religieux à la gloire de Notre Dame tous les samedis ; celle-ci saura bien, alors, le récompenser d'une telle discipline et d'une telle dévotion ${ }^{53}$. Le bourgeois naturellement obéit, et au bout d'un an de loyal service, comme promis et prévu, Notre Dame lui apparaît en songe, en lui ordonnant de faire tailler dans le plus grand secret une statue de la Vierge avec l'enfant Jésus. Il aura alors en retour

50. La formule est calquée sur celle que Michel Serres a appliquée au fonctionnement de la statue du Commandeur dans le mythe de Don Juan (voir Michel Serres, Hermès I - La Communication, Paris, 1968, p. 233).

51. Selon Gabriel Bermain, les statues représentent des êtres agissant pour leur compte aussi. C'est cette croyance que d'ailleurs Lucien met en scène satiriquement dans son Philopseudès (voir Gabriel Germain, "Du sacré au fantastique ", dans Bulletin de l'Association Guillaume Budé, t. 4 (décembre 1968), p. 461-471 ; Nilsson Martin Persson, Geschichte der griechischen Religion, Munchen, 3. Durchgesehene une erganzte Aufl., t. I, 1974, p. 74.)

52. Lecoy, éd. cit., v. 8514-8531.

53. Ibid., v. $8540-8592$. 
un don de sa part ${ }^{54}$. Seulement, le texte nous dit que le pape avait interdit, quelque temps auparavant, l'édification de toute statue représentant les apparences d'homme ou de femme, pour mettre un frein aux fausses croyances et à l'adoration des idoles païennes ${ }^{55}$ - le récit met donc en scène le conflit épistémologique -, et il avait fait réunir celles existantes dans un dépôt appelé "Colosé 》, à l'endroit même où notre bourgeois s'était uni d'intention avec la mystérieuse statue ${ }^{56}$. Pour paraphraser un des romans d'Oscar Panizza mis à l'index il y a un peu plus d'un siècle, on pourrait dire que le bourgeois, appelé à choisir entre l'autorité de l'immaculée conception de la Vierge et celle de l'immaculée conception du pape, tient à sa tête de personnage, et décide qu'il faut prendre d'abord conseil auprès du pape. Le pape naturellement ne peut modifier sa décision à cause du rêve d'un pauvre malheureux ${ }^{57}$. Cependant, Notre Dame apparaît la nuit suivante une deuxième fois au bourgeois, en lui reprochant de ne pas reconnaître son autorité, et en le menaçant de perdre son don ${ }^{58}$. Le pape invite alors prudemment le jeune homme à attendre une troisième apparition ${ }^{59}$. La nuit suivante, la Vierge se manifeste à nouveau, et dans un discours d'une violence surprenante chez la douce mère du Christ, elle accable le pauvre jeune homme de tous les reproches, et le rend responsable de tous les malheurs qui arriveront dès lors au genre humain, à cause de son refus ${ }^{60}$. $\mathrm{Au}$ réveil, l'homme va voir le pape, décidé à le pcrsuader de faire construire une très belle statue de la Madone, couverte d'or et d'argent. C'est cette statue qui est exposée à l'admiration et à l'adoration des

54. Ibid., v. 8604-8627.

55. Le culte des images sacrées au Moyen Âge a pour fonction de s'opposer à la pratique païenne de l'idolâtrie, mais en même temps l'excès de vénération associé à la nature anthropomorphe de l'iconographie éveille des soupçons. C'est que "l'image médiévale 'présentifie' sous les apparences de l'anthropomorphe et du familier, l'invisible dans le visible, Dieu dans l'homme, l'absent dans le présent, le passé ou le futur dans l'actuel " (Jean-Claude Schmitt, «Images », dans Jacques Le Goff et Jean-Claude Schmitt, Dictionnaire raisonné de l'Occident médiéval, Paris, 1999, p. 501).

56. Ibid., v. 8305-8341.

57. Ibid., v. 8635-8648.

58. Ibid., v. 8652-8661.

59. Ibid., v. 8663-8669.

60. Ibid., v. 8670-8693. 
fidèles sur le parvis de l'église de Notre-Dame de la Rotonde à Rome ${ }^{61}$. Quant au bourgeois, il continue avec les mêmes foi et abnégation son abstinence et son service du samedi, jusqu'au jour où la statue disparaît sous les yeux des fidèles qui écoutent la messe, au plus grand désespoir du pauvre jeune homme qui, en pleurs, adresse une très belle prière d'amour marial à Notre Dame ${ }^{62}$. C'est alors que la statue réapparaît avec la main droite bien fermée, montrant l'anneau du bourgeois. Sur le conseil du pape, le bourgeois ose alors demander la restitution de son anneau à la statue de la Vierge qui le lui rend, mettant fin à sept ans de tourments, et décidant, par là même, saint Grégoire à faire refleurir le culte des images sacrées ${ }^{63}$.

Or, le terme " image " ${ }^{64}$, qui est utilisé en ancien français pour désigner une statue, possède une forte charge d'ambiguïté épistémologique et ontologique, y compris pour un lecteur médiéval. Le chrétien qu'est tout lecteur médiéval sait qu'il est écrit dans la Bible : "Tu ne feras point d'image taillée ni de représentation quelconque des choses qui sont en haut dans les cieux, qui sont en bas sur la terre, et qui sont dans les eaux plus bas que la terre 65 ». Mais il sait aussi que l'on peut lire dans la Bible des Septante que les dieux de

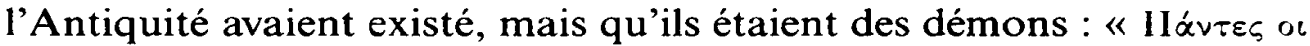

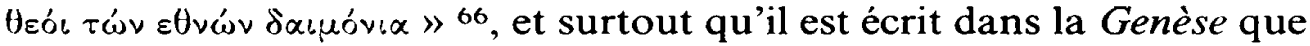

61. Ibid., v. 8694-8735.

62. Ibid., v. 8736-8781.

63. Ibid., v. 8784-8820.

64. Comme on le sait, le mot "ymage " est utilisé au Moyen Âge pour indiquer des statues représentant des divinités par la sculpture (voir $\mathrm{La}$ Vie de saint Alexis, éd. Christofer Storey, Genève et Paris, 1968, p. 101, XXXIV, 168, "Deus fist l'imagine pur sue amour parler »). Mais son champ lexical est plus vaste et ambigu, et il recouvre dans sa fécondité tous les sens du terme latin imago. Que l'on pense, par exemple, à l'utilisation qu'en fait Guillaume de Machaut dans son Voir Dit, lorsque le je rêve de la « douce ymage » de sa dame, détournant sa tête et son visage.

65. Exode, xx, 4.

66. Bible des Septante, Ps. 95 (96), v. 5. Par ailleurs, chez les Romains, on connaissait des statues qui versaient des larmes, ou qui transpiraient du sang (voir Julius Obsequens, Prodigia, dans Tite-Live, $A$ h urhe condita, trad. Alfred C. Schlesinger, Cambridge, London, t. 14 [1987], p. 249-250 A.U.C. 591, et 269-270 A. U.C. 640). Chez les Grecs aussi, il existe des statues qui transpirent, comme celle d'Hector dans la ville d'Ilion (voir Philostrate, Hpwixós, dans 
Yahvé avait façonné l'homme «ad imaginem et similitudinem nostram $\gg 6$ ?.

Cependant c'est l'interdit de l'Exode qui est repris dans le texte, où il est rappelé à deux reprises que le pape avait fait détruire ou disparaître toute statue représentant des corps humains ${ }^{68}$.

Que représentent-elles, alors, par-delà le but avoué de convertir, à travers le miracle de la disparition-réapparition de la statue de Notre Dame, un grand nombre de mécréants ${ }^{69}$, ces statues et ces visions?

Ces différentes images, celle de la statue de la divinité païenne, celle de Notre Dame en rêve, celle de la statue de Notre Dame, semblent avoir en commun beaucoup de traits différentiels, au point qu'il peut sembler difficile de les distinguer. Or, si l'on se réfère à des personnages ou à des récits dans lesquels la statue est le simulacre de l'être ${ }^{70}$, les images de notre récit semblent être des avatars du personnage du deus

Opera, éd. C. L. Kaiser, Lipsiae, t. II, 1870, p. 181-182). Plus en général, les Grecs croyaient que certaines statues étaient d'origine divine : achiropoï̀tes, c'est-à-dire « non faites de main d'homme ».

67. Genèse, I, 26.

68. Mais, comme l'on sait, la réalité historique est plus complexe, puisque le pape Grégoire le Grand considérait les images comme la "Bible des illettrés ». C'est dans une lettre écrite en l'an 600, et destinée à l'évêque de Marseille, Serenus, qui avait fait détruire dans son diocèse toutes les peintures, pour ne pas attiser le feu de l'idolâtrie, que Grégoire le Grand résume la fonction pastorale et religieuse que celles-ci doivent avoir pour un bon chrétien : 1) rappeler l'histoire sainte ;2) inciter le fidèle à la contrition et au repentir ; 3) instruire les illettrés sur le sens des Écritures. Leur corporéité doit de fait rappeler la similitude entre Dieu et l'homme, sans jamais inciter à l'adoration. Mais le Moyen Âge a connu cette lettre dans une autre version, qui est en réalité le résultat de l'interpolation dans la première d'une deuxième lettre de Grégoire le Grand destinée, celle-ci, à un ermite, Secundinus, lui demandant des reliques et une image du Christ. Or, le pape, tout en estimant que ce désir de l'ermite peut être comparé à celui, profane, d'un amoureux épiant la femme qu'il aime - donc condamnable --, consent à la requête de Secundinus, reconnaissant de fait la légitimité, sinon la nécessité, d'une symbolique religieuse accompagnant la dévotion, et jetant un pont entre affection et foi, entre immatérialité de l'ubiquité divine et matérialité de la mortalité humaine.

69. Lecoy, éd. cit., v. 8788-8791.

70. Dans l'Égypte antique, par exemple, le sculpteur est «celui qui fait vivre ». 
ex-machina, réifications plutôt qu'incarnations de l'éternel féminin, qui dérèglent les automatismes narratifs, se transforment en golem au féminin ${ }^{71}$ de l'être de papier ou de parchemin.

Cette matière, à laquelle le bourgeois-lecteur médiéval se lie par le mariage, est celle de l'illusion référentielle, du mythe ${ }^{72}$, qui piège le lecteur en même temps qu'elle fait exploser l'intenable tenue narrative d'un corps et d'un esprit sans entrailles, d'une image de papier.

La matière, avec son immuabilité et sa constance à soi référentielle, s'oppose symboliquement et trompeusement à la condition purement verbale du corps des personnages, en même temps qu'elle se pose comme construction factice et iconoclaste de leur désir fictionnel de s'incarner dans un corps pour toujours. Le corps, de cire, de pierre ou de cuivre, est alors perçu comme évoluant à l'extérieur du texte fictionnel, et cependant encore à l'intérieur de la condition verbale, celle de l'interprétation : il est à la fois représentation du désir objectal du personnage et du désir narcissique de l'auteur.

C'est l'illusoire, illusoire à la fois du lecteur à la recherche de l'insoutenable légèreté du personnage de papier, et illusoire d'une conscience, celle de l'autcur, confinée dans la non-contradiction du cahier de charges du personnage, qui alimente ce mimétisme de l'incarnation par l'aliénation. Le corps statuaire devient alors une construction factice et iconoclaste de deux désirs, de deux formes de libido en

71. Le parallèle peut paraître sans doute impropre, mais il me semble que le golem, simulacre d'homme que, d'après la tradition, au XvI ${ }^{\mathrm{e}}$ siècle, un rabbin polonais ou pragois aurait modelé en se servant d'argile, et auquel il aurait donné la vie en lui apposant sur la bouche (ou la tête) un papier avec le texte d'un verset de la Bible, possède les mêmes caractéristiques sémiotiques que la statue, à qui l'acte de parole a donné les apparences d'un vrai-faux personnage romanesque (voir, par exemple, à propos de la représentation du golem, "Isabella d'Égypte ", dans Romantiques allemands, Paris, Bibliothèque de la Pléiade, t. II, 1973, cité dans Pierre Jourde et Paolo Tortonese, Visages du Double, Paris, 1996, p. 172-173).

72. Le motif du mariage avec la statue n'est en fin de compte qu'une variante du mythe de la hiérogamie, qui est au Moyen Âge récupéré par les écrivains chrétiens, et intégré dans les vies des saints. Ainsi, dans la Légende Dorée de Jacques de Voragine, sainte Agnès est attachée et mariée au Christ par l'anneau que celui-ci a mis à la main droite de la jeune vierge. Mais la hiérogamie est aussi, comme tout mythe, un motif littéraire. 
conflit : d'une part, le désir humain de l'auteur de se disperser dans l'immuabilité du verbe, sa libido verbis, d'autre part, celui surhumain de sa conscience de se réifier dans une image qui, comme il est écrit dans la Bible, ne pourra que fondre " comme de la cire devant ta face " ${ }^{73}$ : sa libido carnis, qui réitère sur un plan fictionnel le mystère de l'Incarnation.

La symbolique de transition du Même à l'Autre, la représentation par une réification d'une chose désirée, même sous une forme algolagnique, et en tant que telle manifestation d'une différenciation ontologique par le dédoublement, semblent être, mutatis mutandis, les mêmes qui poussent, à une autre époque, le personnage de Mélück Maria Blainville à piéger l'âme et le destin d'un personnage, auquel l'auteur Ludwig Achim von Arnim ${ }^{74}$ a décidé d'attribuer le nom de Saintrée, c'est-à-dire du protagoniste du roman d'Antoine de la Sale, auquel je faisais référence au début. Ces personnages, ou, comme les définit André Breton, " ces objets parfaits d'illusion, poussant leur coquetterie jusqu'à se soustraire à la volonté de l'auteur ${ }^{75}$, semblent structurer et formaliser le stream of consciousness de l'auteur. Mannequins 76 ou statues, ils possèdent dans leur bagage encyclopédique de personnages, à la fois la conscience d'un manque et d'une supériorité : l'absence d'une dialectique ontologique, du doute qu'insuffle l'« aporétique du temps " ${ }^{7}$, mais aussi le destin d'une tenue esthétique. Ils savent, comme le rappelle Horace, que "Verbo sicut mollissimam

73. Le Livre de Judith, 16, 18.

74. Voir Ludwig Achim von Arnim, Mélück Maria Blainville, trad. Françoise Rétif, Paris, 1996, p. 39-89. L'esprit et l'âme de Saintrée sont piégés par Mélück dans un mannequin qui reproduit l'image de celui-ci. Lorsque celui-ci trahira l'amour de Mélück pour se marier avec sa fiancée, le mannequin absorbera toute son énergie vitale, en devenant une image du personnage Saintrée, en même temps que celui-ci dépérira.

75. André Breton, "Introduction ", dans Ludwig Achim von Arnim, Contes bizarres, trad. Théophile Gautier fils, Paris, 1964, p. 16.

76. Dans la nouvelle d'Arnim, le mannequin se refuse à rendre l'habit avec les larmes de la future épouse de Saintrée, Mathilde, que celui-ci avait offert en gage d'amour à Mélück. Tout comme la statue recourbe le doigt, le mannequin-automate croise les bras et ne les desserre plus (voir Arnim, op. cit., p. $51-52,68$.)

77. Paul Ricour, Temps et récit. 3. Le temps raconté, Paris, 1985, p. 313. 
ceram $"{ }^{78}$, mais aussi que verbe et matière ne sont plus qu'un dansl'illusion référentielle d'une " image-chose " ${ }^{79}$.

La projection du discontinu littéraire sur le continu réel, qu'ils incarnent ou réifient, est pour le lecteur une des conditions de l'intelligibilité du monde : c'est-à-dire de la matière et de l'esprit, de l'essence et de l'existence. L'illusion référentielle du corps et de l'esprit, c'est-à-dire de l'épistémè du monde possible, sert ainsi de tain aux apories et aux anachronismes du monde réel. Elle alimente la contradiction vitale entre corps et parole, entre vérité historique et vérité artistique, entre nos paroles d'aujourd'hui et ces corps d'antan, mais aussi entre ces paroles médiévales et nos corps modernes.

Claudio GALDERISI.

78. Cité in Totius Latinitatis Lexicon Aegidi Forcellini, Prati, typis Aldinianis, t. II, 1861, «cera».

79. Pour Jean-Claude Bonne, la choséité de l'image, c'est l'essence même de la matière, sa forme préfigurative, qui ne peut être sémantisée, et qui cependant a une fonction évocatoire très forte (voir Jean-Claude Bonne, Jean-Claude Schmitt, Daniel Barbu et Jérôme Baschet, «Images médiévales ", dans Annales : Histoire, Sciences sociales, 1 [1996], p. 3-133). 